\title{
sciendo
}

\section{Ending MMA Combat, Specific Grappling Techniques According to the Type of the Outcome}

\author{
by \\ Fábio dal Bello ${ }^{1,5}$, Ciro José Brito², John Amtmann³, Bianca Miarka2,4
}

\begin{abstract}
This study compared grappling motor actions of male mixed martial arts (MMA) athletes considering outcome types from Ultimate Fighting Championship (UFC) bouts. A validated protocol of technical-tactical analysis was utilized as in previous studies addressing MMA performance analysis, and Kruskall Wallis and U Mann-Whitney tests were applied to compare effects of types of outcome decisions (Split vs. Unanimous Decision vs. Knockout-KO/Technicalknockout-TKO vs. Submission). Unanimous Decision showed higher frequencies of takedowns attempted/round than KO/TKO and Submission outcomes ( $p \leq 0.05 ; 1.9 \pm 1.9$ vs. $1.3 \pm 1.4$ vs. $1.0 \pm 1.1$ attempts). Bouts with Split Decision demonstrated higher takedowns/round than bouts ended by Submission ( $p=0.048 ; 0.4 \pm 0.7$ vs. $0.2 \pm 0.6$ attempts). TKO/KO showed lower values of sweeps/round ( $p=0.008,0.0 \pm 0.0$ vs. $0.1 \pm 0.3$ attempts) and takedowns attempted/round ( $p=0.014,1.3 \pm 1.4$ vs. $2.0 \pm 1.6$ attempts) than bouts ending by Split Decision. The Submission outcome showed a higher frequency of submissions attempted/round than KO/TKO and Unanimous Decision ( $p \leq 0.041$, $0.3 \pm 0.7$ vs $0.2 \pm 0.5$ vs $0.2 \pm 0.5$ ). These results show a large specificity in the type of grappling attack/situation according to the strategy to end the combat. These results also show that the grappling strategy and tactics are variable depending on the strengths and weaknesses of the athletes, and can be used by coaches and athletes to develop specific training programs.
\end{abstract}

Key words: time and motion studies, task performance and analysis, martial arts, motor control, high-intensity interval training, teaching.

\section{Introduction}

Mixed martial arts (MMA) can be separated into striking (Slimani et al., 2017; Tabben et al., 2018) and grappling combat technical actions (del Vecchio et al., 2011; Miarka et al., 2017). Technicaltactical analysis is a way to understand the factors explaining success in elite-level MMA combats (James et al., 2017; Miarka et al., 2016a; Santos et al., 2018). Recently studies indicated that successful grappling technical-tactical actions in MMA come from two main combat sports: Brazilian Jujitsu (BJJ) and wrestling (James et al., 2017; Miarka et al., 2016a, 2016b). Although grappling fundamentals are commonly accepted as essential to success in MMA, little is known about technical demands with specific actions (i.e. arm triangle choke, shoulder lock, knee bar, ankle lock or other isolated skills) (Kirk et al., 2015) and tactical demands (i.e. combat situation, as standing up or groundwork combat or/and specific decisions/conditions depending on internal and external factors) (Antoniettô et al., 2019; Fernandes et al., 2019; Ghoul et al., 2017; James et al., 2017; Miarka et al., 2016a; Maszczyk et al., 2018). A full literature review yielded no results quantifying grappling

1 - Head of Physical Activity and Sports Science Master Program, Universidad Santo Tomás, Santiago, Chile.

2 - Laboratory of Physiological and Motor Analysis in Health and Performance of the Physical Education Department, Physical

Education Department, Federal University of Juiz de Fora, Minas Gerais, Brazil.

3 - Safety, Health and Industrial Hygiene Department, Montana Tech of the University of Montana, Montana, United States.

4 - Fight Department, School of Physical Education and Sport, Federal University of Rio de Janeiro, Rio de Janeiro, Brazil.

5-Universidad Politécnica de Madrid, Madrid, Spain

Authors submitted their contribution to the article to the editorial board.

Accepted for printing in the Journal of Human Kinetics vol. 67/2019 in June 2019. 
actions in MMA. Recent research revealed crucial differences between outcomes regarding the methods applied during standing actions of female MMA athletes with the most bouts ending by Unanimous and Split Decisions (Antoniettô et al., 2019; Miarka et al., 2016a). In male athletes, Hutchison et al. (2014) and James et al. (2017) identified factors that were associated with a higher incidence of Knockout (KO) or Technicalknockout (TKO) including age, weight, fight significance, time within the round, and time since the last combat. Consequently, tactical analysis is one of the basic elements for developing sportspecific training and/or proper conditioning programs to the requirements of the combat methods in MMA athletes.

Although, these studies provided important information for athletes and coaches, we believe that MMA athletes require specific analysis of technical-tactical actions associated specifically with grappling actions (Miarka et al., 2016a, 2017, 2017a). These actions require a diverse skillset, including stand-up grappling and ground grappling skills because relationships between these skills represent the primary aspects of grappling attack systems (Coswig et al., 2016; del Vecchio et al., 2011; Sterkowicz-Przybycien et al., 2016). In fact, a previous study indicated that technical-tactical actions differ between winners and losers (Miarka et al., 2016b), but no studies investigated specific grappling technical-tactical action differences and outcomes of the bout.

The analysis and diagnosis of grappling technical-tactical actions specific to MMA can provide essential information to MMA coaches and athletes that can be used to enhance performance during MMA competition (Brandt et al., 2018; Coswig et al., 2016; Karpman et al., 2016; Miarka et al., 2014, 2015). Moreover, evaluation of the grappling technical actions (i.e. takedowns, takedown shots, upper and lower body takedowns, chokes, rear naked choke, arm triangle, arm bar, ankle lock, arm lock, and others) has not been performed in male bouts (Brito et al., 2017; Miarka et al., 2018). Such analysis will show the variety of strategies and tactics used by MMA outcomes, and may provide coaches and athletes with an opportunity to make other choices during training and competition. This combat sport is very complex with various grappling strategies over five minutes per round involving acyclic open tasks, and the identification of differences as a potential mediator of success could help to understand how technical-tactical actions affect outcomes in order to quickly adapt to grappling situations during the round (Miarka et al., 2017a; Gronek et al., 2015; Maszczyk et al., 2018). Thus, this study compared grappling motor actions of male MMA athletes considering different outcomes (Submission, $\mathrm{KO} / \mathrm{TKO}$, Unanimous and Split Decision,) from Ultimate Fighting Championship (UFC) bouts, providing practical implications for MMA training. We hypothesized that grappling actions would differ between outcomes.

\section{Methods}

\section{Participants}

The sample was composed of 304 UFC rounds divided into $\mathrm{KO} / \mathrm{TKO}(\mathrm{n}=54)$, Submission $(\mathrm{n}=40)$, Split $(\mathrm{n}=40)$ and Unanimous Score Decision $(\mathrm{n}=170)$, from 58 events taking place within the 2012-2014 period (TUF Finale 16-19; UFC 152-174, Fight Night 28-50; UFC on Fox 3-11; UFC on FUEL TV 4-7). A minimum of six weeks of rest was observed between bouts to prevent stress interference (James et al., 2013). All participants had previous experience with professional UFC events, rules and procedures. No interferences were made in the training process, nutritional or hydration status and the athletes maintained the weight loss recovery time 24 hours between official weigh-in and the bout (Brandt et al., 2018; Coswig et al., 2018; Jetton et al., 2013; Matthews and Nicholas, 2016). The inclusion criteria considered only bouts with three-rounds, including knockout $(\mathrm{KO})$, technical knockout (TKO), submission and score decisions (split and unanimous), and we excluded bouts with more than three rounds and/or with characteristics that disqualified prospective outcomes comparisons - bouts which finished in "draw" or "no contest".

This study was submitted to and approved by the Local Committee of Ethics in Research, following the rules of resolution 196/96 of the National Health Council. The present study ensured anonymity and confidentiality by replacing the athletes' personal identification; furthermore, there are no ethical issues in analyzing or interpreting data obtained at public events, as established by previous protocols (Miarka et al., 2014, 2015). 


\section{Measures}

This is a comparative and descriptive applied study. Using a technical-tactical protocol identified in previous studies addressing MMA performance analysis (Miarka et al., 2015, 2016a), we determined the specific grappling actions analyzed by referee's decision. This information brings new concepts of performance evaluation and training programs considering the specific combat phase of each outcome. In sequence, the data were collected from professional UFC events taking place at air-conditioned arenas, except the Ibirapuera's Gym, between 18:30 and 24:00 with temperature ranging between 24.5 and $27.0^{\circ} \mathrm{C}$. Afterwards, in the very last stage, we compared types of outcomes (TKO/KO, Submission, Unanimous and Split Score Decision) to verify the impact of each technical-tactical grappling action. Design and Procedures

Grappling actions were subdivided into four categories according to the movement pattern applied, including Stand up determinant actions (i.e. lower body takedowns attempted and landed, stand up attempts, sweeps, takedown shots attempted and landed, upper body takedowns attempted and landed), and determinant groundwork actions (i.e. advances from back, halfguard, mount and side, advances to back, offensive guard passes, successful submission, ankle lock attempted, arm bar attempted, knee bar attempted, shoulder lock attempted and other locks attempted, guillotine choke attempted, rear naked choke attempted, triangle choke attempted, arm triangle choke attempted and other chokes attempted) (Kirk et al., 2015). Table 1 shows percentages of all grappling actions analysed per frequency by round.

Technical-tactical action changes were observed by five researchers, according to their frequency, following a previously established protocol (Miarka et al., 2017). In order to guarantee ecological validity and to verify the elite status of the sample, the bouts were analyzed by the FightMetric Team using UFC records of professional quality and recorded by performance analysts. All available videos of sufficient quality (standard definition 480/60i) and taken from a landscape view of the entire competition area were included in the analysis (Sterkowicz-Przybycien et al., 2016). The reliability between measurements obtained for each variable was verified with the
Intraclass coefficient of correlation (ICC); takedown attempts (CCI $=0.92,95 \%$ I.C. 0.87 to $0.97 ; p \leq 0.001)$, submission attempts (CCI $=0.936$; $95 \%$ I.C. 0.871 to $0.969 ; p \leq 0.001)$, Lock attempts $(\mathrm{CCI}=0.90,95 \%$ I.C. 0.80 to $0.95 ; p \leq 0.001)$, choke attempts (CCI $=0.83$; $95 \%$ I.C. 0.68 to $0.92 ; p \leq$ 0.001 ); Mount advances (CCI $=0.74,95 \%$ I.C. 0.52 to $0.87 ; p \leq 0.001)$; Back advances (CCI $=0.50,95 \%$ I.C. 0.19 to $0.73 ; p \leq 0.001$ ) and side advances (CCI $=0.80,95 \%$ I.C. 0.62 to $0.90 ; p \leq 0.001)$.

\section{Statistical Analysis}

The Kolmogorov-Smirnov test (K-S) was used to determine data's normal distribution. Descriptive data of frequency of dependent variables are presented as median, first quartile (1Q) and third quartile (3Q). For these nonparametric data, Kruskall Wallis and Bonferroni post hoc tests were applied to compare effects of types of outcome decisions (Split vs. Unanimous Score Decision vs. KO/TKO vs. Submission). Afterwards, the effect size for non-parametric analysis was calculated, defined as $\mathrm{ES}=\mathrm{Z} / \mathrm{VN}$, where ES represented the effect size, $Z$ was derived from the conversion of the Wilcoxon test, and $\mathrm{N}$ was the total number of observations. The analysis considered ES-values as small (ES $<0.10)$, medium $(E S<0.30)$ or large effect size $(E S<0.50)$. The significance level of $p \leq 0.05$ was used. All analyses were conducted using SPSS 20.0 for Windows.

\section{Results}

Table 2 presents standing combat grappling actions that occurred during MMA rounds.

Regarding standing combat actions, MMA bouts ending with a Unanimous Decision showed higher frequencies of takedowns attempted/round than $\mathrm{KO} / \mathrm{TKO}(p=0.05, \mathrm{ES}=0.13)$ and Submission outcomes $(p=0.018$, ES $=0.17)$. Mainly, Decision Unanimous showed higher Takedowns Attempted/round than Submission $(p=0.015$, ES = 0.17). Split Decision showed higher values of sweeps/round $(p=0.010, \mathrm{ES}=0.27)$ and takedowns attempted/round $(p=0.014, \mathrm{ES}=0.25)$ than bouts ended by TKO/KO. KO/TKO showed lower frequency of sweeps/round $(p=0.010, \mathrm{ES}=0.27)$ than Submission outcomes. 
Table 1

Percentages of all grappling actions analysed by frequency by round.

\begin{tabular}{|c|c|c|c|c|c|c|c|c|c|}
\hline Frequencies & 0 & 1 & 2 & 3 & 4 & 5 & 6 & 7 & 8 \\
\hline \multicolumn{10}{|l|}{ Stand up actions } \\
\hline Takedowns Landed (\%) & 59.1 & 24.4 & 9.4 & 5.8 & 1.3 & & & & \\
\hline Takedowns Attempted (\%) & 33.4 & 22.1 & 17.9 & 11.4 & 6.8 & 4.5 & 2.9 & 0.3 & 0.6 \\
\hline Takedowns Slams (\%) & 92.5 & 6.2 & 1.3 & & & & & & \\
\hline Offensive Passes (\%) & 70.5 & 13.3 & 8.1 & 4.2 & 1.3 & 2.3 & 0.3 & & \\
\hline Successful Submission $(\%)$ & 98.7 & 1.3 & & & & & & & \\
\hline Takedown Shots Landed (\%) & 80.5 & 14.3 & 4.2 & 1.0 & & & & & \\
\hline Takedown Shots Attempted (\%) & 53.2 & 22.1 & 13.6 & 6.5 & 1.3 & 2.6 & 0.6 & & \\
\hline Takedown Shots Slams (\%) & 97.7 & 1.9 & 0.3 & & & & & & \\
\hline L.B. Takedowns Landed (\%) & 78.9 & 17.9 & 2.6 & 0.6 & & & & & \\
\hline L.B. Takedowns Attempted (\%) & 63.3 & 22.4 & 9.7 & 2.9 & 1.3 & 0.3 & & & \\
\hline L.B. Slams (\%) & 95.8 & 3.6 & 0.6 & & & & & & \\
\hline U.B. Takedowns Landed (\%) & 94.5 & 4.9 & 0.3 & 0.3 & & & & & \\
\hline U.B. Takedowns Attempted (\%) & 92.2 & 7.1 & 0.3 & 0.3 & & & & & \\
\hline U.B. Slams (\%) & 98.7 & 1.3 & & & & & & & \\
\hline \multicolumn{10}{|l|}{ Groundwork actions } \\
\hline Submissions Attempted (\%) & 85.7 & 10.4 & 3.2 & 0.3 & 0.3 & & & & \\
\hline Chokes Attempted (\%) & 87.7 & 9.4 & 2.6 & 0.3 & & & & & \\
\hline Locks Attempted (\%) & 97.1 & 2.3 & 0.3 & 0.3 & & & & & \\
\hline Rear Naked Choke Attempted (\%) & 95.8 & 3.2 & 1.0 & & & & & & \\
\hline Guillotine Choke Attempted (\%) & 93.5 & 5.5 & 0.6 & 0.3 & & & & & \\
\hline Triangle Choke Attempted (\%) & 99.4 & 0.6 & & & & & & & \\
\hline Arm Triangle Choke Attempted (\%) & 99.0 & 1.0 & & & & & & & \\
\hline Other Choke Attempted (\%) & 99.0 & 1.0 & & & & & & & \\
\hline Arm Bar Attempted (\%) & 98.7 & 1.3 & & & & & & & \\
\hline Shoulder Lock Attempted (\%) & 99.0 & 0.6 & 0.3 & & & & & & \\
\hline Ankle Lock Attempted (\%) & 99.0 & 1.0 & & & & & & & \\
\hline Knee Bar Attempted (\%) & 100.0 & & & & & & & & \\
\hline Other Lock Attempted (\%) & 100.0 & & & & & & & & \\
\hline Advance To Half-Guard (\%) & 83.1 & 12.7 & 3.2 & 1.0 & & & & & \\
\hline Advance To Side (\%) & 86.7 & 9.7 & 2.9 & 0.6 & & & & & \\
\hline Advance To Mount (\%) & 94.2 & 4.9 & 1.0 & & & & & & \\
\hline Advance To Back(\%) & 89.6 & 6.8 & 3.2 & 0.3 & & & & & \\
\hline Advance From Half-Guard (\%) & 92.2 & 6.8 & 0.6 & 0.3 & & & & & \\
\hline Advance From Side (\%) & 96.4 & 2.3 & 1.3 & & & & & & \\
\hline Advance From Mount (\%) & 96.1 & 3.6 & 0.3 & & & & & & \\
\hline Advance From Back (\%) & 95.5 & 3.6 & 1.0 & & & & & & \\
\hline Stand Ups (\%) & 64.9 & 24.4 & 8.1 & 2.3 & & & & & \\
\hline Sweeps (\%) & 93.8 & 6.2 & & & & & & & \\
\hline
\end{tabular}


Table 2

Standing combat grappling actions observed during MMA rounds, separated by outcome types.

\begin{tabular}{|c|c|c|c|c|c|c|}
\hline \multirow{2}{*}{$\begin{array}{c}\text { Stand up Grappling } \\
\text { Action }\end{array}$} & \multirow{2}{*}{$\begin{array}{l}\text { Unanimous } \\
\text { Decision } \\
\operatorname{Med}(1 \mathrm{Q} ; 3 \mathrm{Q})\end{array}$} & \multirow{2}{*}{$\begin{array}{c}\text { Split } \\
\text { Decision } \\
\operatorname{Med}(1 \mathrm{Q} ; 3 \mathrm{Q})\end{array}$} & \multirow{2}{*}{$\begin{array}{l}\text { Submission } \\
\operatorname{Med}(1 \mathrm{Q} ; 3 \mathrm{Q})\end{array}$} & \multirow{2}{*}{$\begin{array}{c}\text { KO/TKO } \\
\operatorname{Med}(1 \mathrm{Q} ; 3 \mathrm{Q})\end{array}$} & \multicolumn{2}{|c|}{ Inferences } \\
\hline & & & & & $\mathrm{X}^{2^{*}}$ & $p$ \\
\hline L.B. Slams & $0.1(0.0 ; 0.1)$ & $0.1(0.0 ; 0.1)$ & $0.1(0.0 ; 0.1)$ & $0.1(0.0 ; 0.1)$ & 3.521 & .318 \\
\hline $\begin{array}{l}\text { L.B. Takedowns } \\
\text { Attempted }\end{array}$ & $0.5(0.0 ; 1.0)$ & $0.5(0.0 ; 1.0)$ & $0.5(0.0 ; 1.8)$ & $0.5(0.0 ; 1.0)$ & 3.506 & .320 \\
\hline $\begin{array}{l}\text { L.B. Takedowns } \\
\text { Landed }\end{array}$ & $0.1(0.0 ; 0.2)$ & $0.3(0.0 ; 1.0)$ & $0.1(0.0 ; 0.2)$ & $0.1(0.0 ; 0.1)$ & 2.507 & .474 \\
\hline Stand Ups & $0.6(0.0 ; 1.0)$ & $0.5(0.0 ; 1.0)$ & $0.5(0.0 ; 1.0)$ & $0.4(0.0 ; 1.0)$ & 5.152 & .161 \\
\hline Foot Sweeps & $0.1(0.0 ; 0.1)$ & $0.1(0.0 ; 0.1)$ & $0.1(0.0 ; 0.1)$ & $0.1(0.0 ; 0.1)$ & 9.168 & .027 \\
\hline $\begin{array}{l}\text { Takedowns } \\
\text { Attempted }\end{array}$ & $1.0(0.0 ; 3.0)^{\mathrm{a}}$ & $2.0(1.0 ; 3.0)^{\mathrm{c}}$ & $1.0(0.0 ; 2.0)$ & $1.0(0.0 ; 2.0)$ & 11.87 & .008 \\
\hline $\begin{array}{l}\text { Takedown Shots } \\
\text { Attempted }\end{array}$ & $1.0(0.0 ; 2.0)^{\mathrm{b}}$ & $0.0(0.0 ; 1.0)^{\mathrm{bc}}$ & $0.0(0.0 ; 1.0)$ & $0.0(0.0 ; 1.3)$ & 8.138 & .043 \\
\hline $\begin{array}{l}\text { Takedown Shots } \\
\text { Landed }\end{array}$ & $0.1(0.0 ; 0.1)$ & $0.3(0.0 ; 1.0)^{\mathrm{b}}$ & $0.1(0.0 ; 0.1)$ & $0.1(0.0 ; 0.1)^{\mathrm{b}}$ & 7.846 & .049 \\
\hline $\begin{array}{l}\text { Takedown Shots } \\
\text { Slams }\end{array}$ & $0.1(0.0 ; 0.2)$ & $0.0(0.0 ; 0.1)$ & $0.1(0.0 ; 0.2)$ & $0.0(0.0 ; 0.1)$ & 1.307 & .728 \\
\hline Takedowns Landed & $0.2(0.0 ; 1.0)$ & $0.3(0.0 ; 1.0)$ & $0.4(0.0 ; 1.0)$ & $0.6(0.0 ; 1.0)$ & 5.891 & .117 \\
\hline Takedowns Slams & $0.0(0.0 ; 0.1)$ & $0.0(0.0 ; 0.1)$ & $0.0(0.0 ; 0.1)$ & $0.0(0.0 ; 0.1)$ & 3.326 & .344 \\
\hline U.B. Slams & $0.0(0.0 ; 0.1)$ & $0.0(0.0 ; 0.1)$ & $0.0(0.0 ; 0.1)$ & $0.0(0.0 ; 0.1)$ & 1.943 & .584 \\
\hline $\begin{array}{l}\text { U.B. Takedowns } \\
\text { Attempted }\end{array}$ & $0.1(0.0 ; 0.1)$ & $0.1(0.0 ; 0.1)$ & $0.1(0.0 ; 0.1)$ & $0.1(0.0 ; 0.1)$ & 4.730 & .193 \\
\hline $\begin{array}{l}\text { U.B. Takedowns } \\
\text { Landed }\end{array}$ & $0.1(0.0 ; 0.1)$ & $0.1(0.0 ; 0.1)$ & $0.1(0.0 ; 0.1)$ & $0.1(0.0 ; 0.1)$ & 1.381 & .710 \\
\hline
\end{tabular}

L.B. - Lower Body; U.B. - Upper Body. ${ }^{*}$ All analyses performed at 3 degree of freedom; $\operatorname{Med}(1 Q ; 3 Q)-\operatorname{Median}\left(1^{\text {st }}\right.$ quartile; $3^{\text {rd }}$ quartile); ${ }^{a} p \leq 0.05$ vs. KO/TKO and Submission; ${ }^{b} p \leq 0.048$ vs. Submission; ${ }^{c} p \leq 0.014$ vs. KO/TKO.

Table 3

Groundwork position observed during MMA rounds, separated by outcome types.

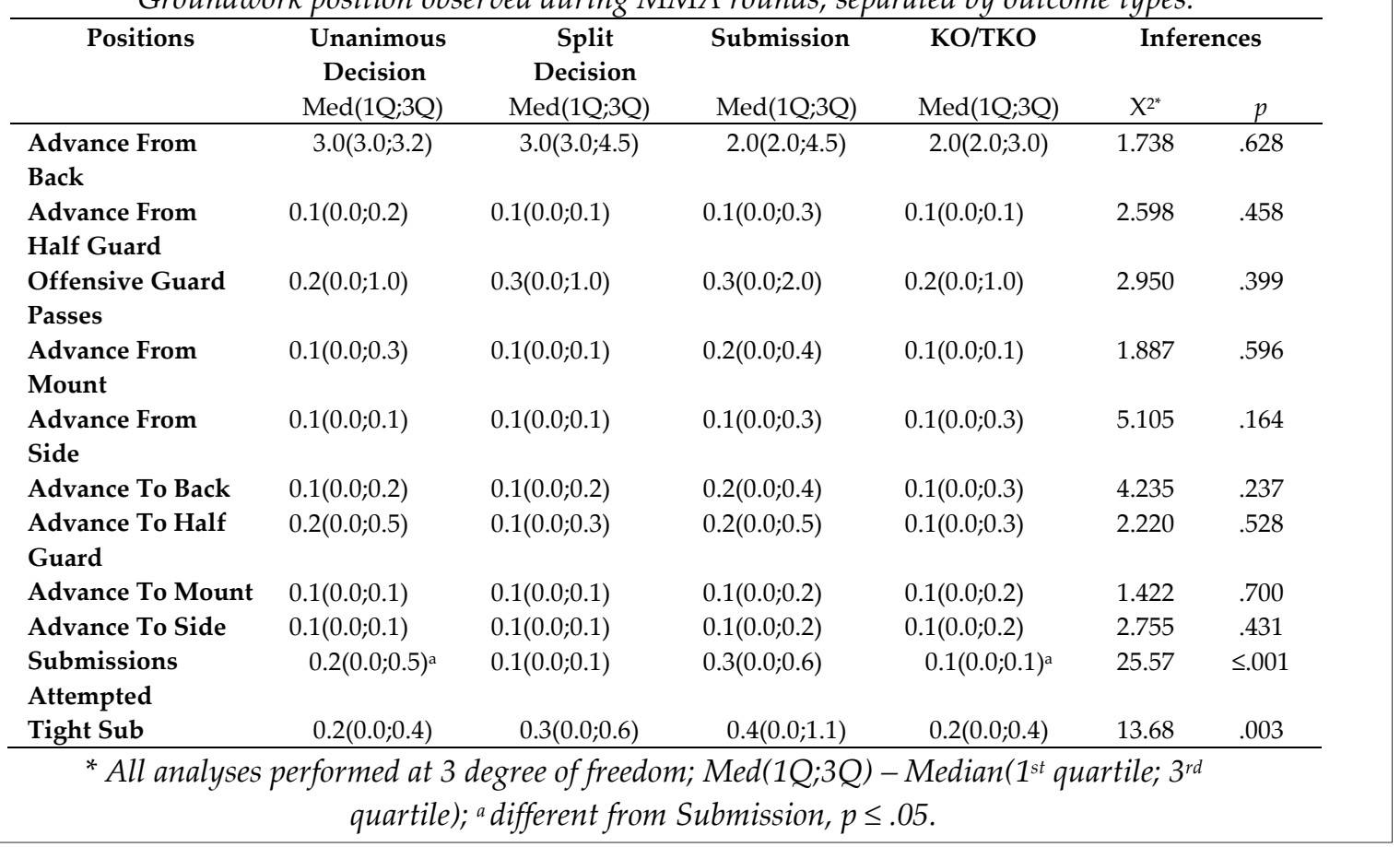


Table 4

Locks and Chokes actions observed during MMA rounds, separated by outcome types.

\begin{tabular}{|c|c|c|c|c|c|c|}
\hline $\begin{array}{c}\text { Locks and } \\
\text { chokes actions }\end{array}$ & $\begin{array}{l}\text { Unanimous } \\
\text { Decision } \\
\operatorname{Med}(1 \mathrm{Q} ; 3 \mathrm{Q})\end{array}$ & $\begin{array}{c}\text { Split } \\
\text { Decision } \\
\operatorname{Med}(1 Q ; 3 Q)\end{array}$ & $\begin{array}{l}\text { Submission } \\
\operatorname{Med}(1 Q ; 3 Q)\end{array}$ & $\begin{array}{c}\text { KO/TKO } \\
\operatorname{Med}(1 Q ; 3 Q)\end{array}$ & Inf & $p$ \\
\hline Ankle Lock & $0.1(0.0 ; 0.2)$ & $0.1(0.0 ; 0.1)$ & $0.1(0.0 ; 0.1)$ & $0.1(0.0 ; 0.1)$ & 2.380 & .497 \\
\hline $\begin{array}{l}\text { Attempted } \\
\text { Arm Bar } \\
\text { Attempted }\end{array}$ & $0.15(0.0 ; 0.2)^{\mathrm{ab}}$ & $0.1(0.0 ; 0.1)$ & $0.1(0.0 ; 0.1)$ & $0.0(0.0 ; 0.1)$ & 11.31 & .010 \\
\hline $\begin{array}{l}\text { Shoulder Lock } \\
\text { Attempted }\end{array}$ & $0.15(0.0 ; 0.3)^{\mathrm{a}}$ & $0.0(0.0 ; 0.1)$ & $0.0(0.0 ; 0.1)$ & $0.0(0.0 ; 0.1)$ & 7.810 & .050 \\
\hline $\begin{array}{l}\text { Total Locks } \\
\text { Attempted } \\
\text { Chokes }\end{array}$ & $0.1(0.0 ; 0.2)^{a}$ & $0.1(0.0 ; 0.2)$ & $0.1(0.0 ; 0.5)$ & $0.0(0.0 ; 0.1)^{a}$ & 9.946 & .019 \\
\hline $\begin{array}{l}\text { Guillotine } \\
\text { Choke } \\
\text { Attempted }\end{array}$ & $0.1(0.0 ; 0.3)^{\mathrm{a}}$ & $0.2(0.0 ; 0.3)$ & $0.3(0.0 ; 0.5)$ & $0.2(0.0 ; 0.4)$ & 7.990 & .046 \\
\hline $\begin{array}{l}\text { Other Choke } \\
\text { Attempted }\end{array}$ & $0.0(0.0 ; 0.1)$ & $0.1(0.0 ; 0.2)$ & $0.0(0.0 ; 0.1)$ & $0.0(0.0 ; 0.1)$ & 1.930 & .587 \\
\hline $\begin{array}{l}\text { Rear Naked } \\
\text { Choke }\end{array}$ & $0.0(0.0 ; 0.2)^{\mathrm{a}}$ & $0.2(0.0 ; 0.4)$ & $0.2(0.0 ; 0.5)$ & $0.1(0.0 ; 0.3)$ & 13.48 & .004 \\
\hline $\begin{array}{l}\text { Attempted } \\
\text { Total Chokes } \\
\text { Attempted }\end{array}$ & $0.1(0.0 ; 0.4)^{a}$ & $0.3(0.0 ; 0.6)$ & $0.3(0.0 ; 0.6)$ & $0.1(0.0 ; 0.3)^{a}$ & 19.75 & $\leq .001$ \\
\hline $\begin{array}{l}\text { Triangle Choke } \\
\text { Attempted }\end{array}$ & $0.0(0.0 ; 0.1)^{\mathrm{a}}$ & $0.0(0.0 ; 0.1)$ & $0.2(0.0 ; 0.3)$ & $0.0(0.0 ; 0.1)$ & 13.24 & .004 \\
\hline $\begin{array}{l}\text { Arm Triangle } \\
\text { Choke } \\
\text { Attempted }\end{array}$ & $0.0(0.0 ; 0.1)$ & $0.1(0.0 ; 0.2)$ & $0.1(0.0 ; 0.1)$ & $0.0(0.0 ; 0.1)$ & 1.930 & .587 \\
\hline
\end{tabular}

In addition, bouts ending in a Split Decision demonstrated higher takedowns attempted/round $(p=0.004, \mathrm{ES}=0.34)$, and takedown shots landed/round ( $p=0.046$, $\mathrm{ES}=0.22$ ) than bouts ending by Submission. Finally, bouts ending in $\mathrm{KO} / \mathrm{TKO}$ demonstrated higher frequency of takedowns landed/round $(p=0.012$, $\mathrm{ES}=0.26)$ than Submissions.

Table 3 demonstrates groundwork grappling positions observed during MMA rounds.

$\mathrm{KO} / \mathrm{TKO}(p=0.043, \mathrm{ES}=0.21)$ and Unanimous Decision ( $p \leq 0.001$, ES $=0.33$ ) showed lower frequencies of submission attempted/round than the Submission outcome. Successful submission presented a significant effect $(p=$ 0.047), however, no difference between groups was observed in the post-hoc test.

Table 4 showed locks and choke actions that occurred during MMA rounds. No frequencies were recorded for Knee Bar Attempted and Other Lock Attempted in analysed bouts.

$\mathrm{KO} / \mathrm{TKO}$ demonstrated lower frequency of chokes attempted/round $(p=0.006$, ES $=0.30)$ and locks attempted/round $(p=0.020, \mathrm{ES}=0.24)$ than Submission. Unanimous Decision showed lower chokes attempted/round ( $p \leq 0.001$, ES=0.29), locks attempted/round $(p \leq 0.001$, ES $=0.18)$, arm bar 
attempted/round $(p=0.005, \mathrm{ES}=0.20)$ and shoulder lock attempted/round $(p=0.036$, ES $=0.15)$ than bouts ended by Submission. In addition, Unanimous Decision showed lower values of arm bar attempted/round $(p=0.047$ ES $=0.14)$ than bouts ended by Split Decision. Unanimous Decision showed a lower frequencies of rear naked choke attempted/round $(p \leq 0.001$, ES $=0.18)$, guillotine choke attempted/round $(p=0.005$, ES $=$ $0.19)$ and triangle choke attempted $(p=0.005, \mathrm{ES}=$ 0.20 ) than bouts ended by Submission.

\section{Discussion}

This study compared grappling motor actions of male MMA athletes considering outcome types, which provides practical implications for MMA training. MMA bouts with Unanimous and Split Score Decision demonstrated higher frequencies of Stand ups/round, Takedowns Attempted and Landed/round than $\mathrm{KO} / \mathrm{TKO}$ and Submission. In addition, KO/TKO demonstrated higher frequencies of Takedown Shots Landed and Lower Body Slams than Submission. In turn, KO/TKO presented a lower frequency of Submissions Attempted/round, Chokes Attempted/round and Locks Attempted/round than Submission. Unanimous Decision showed lower Submissions Attempted/round lower Advance from Side Attempted/round, and Chokes Attempted/round, Locks Attempted/round, Arm Bar Attempted/round, Shoulder Lock Attempted/round, Rear Naked Choke Attempted/round, Guillotine Choke Attempted/round and Triangle Choke Attempted than bouts ending by Submission. A schematic comparison between outcome types of performance for MMA athletes may be helpful to observe differences related to the specific situations and technical-tactical actions (Miarka et al., 2016a). Furthermore, data of different situations can offer crucial information to improve personalized and contextualized training plans with potentially unknown key factors (Coswig et al., 2016). The present study, to the best of our knowledge, was the first to investigate technicaltactical actions considering different outcomes in male MMA athletes.

In 174 bouts, $16 \%$ ended in either $\mathrm{KO}$ or TKO, $11.4 \%$ by submission and $72.4 \%$ by judges' decision. The mechanism of contact resulting in a
$\mathrm{KO}$ was predominantly a direct blow to the head in standing and groundwork combats (Miarka et al., 2016a). In contrast, del Vecchio et al. (2011) showed that most of the male amateur matches ended in $\mathrm{KO}(57.7 \%)$. Based on bouts held at the highest level, i.e., the UFC, it has been demonstrated that time and grappling play a dominant role in the sport of MMA (James et al., 2017; Miarka et al., 2019; Miarka et al., 2017a) considering performance of such MMA athletes as Ronda Rousey (judo), TJ Dillashaw (wrestling), Demetrius Johnson (wrestling), and Cain Velasquez (wrestling) - all of whom were champions in their respective weight classes. These grappling athletes have not been strikers for a long time, but it did not take them long to learn boxing and muay thai techniques which they incorporated into their arsenal (Miarka et al., 2016a). Although all of these athletes come from a grappling background, there are many strategic differences between them, some aim at technical knockouts/knockout, others try locks or chokes submissions, and there are still those who prefer to try to score the most throughout the combat period, which can lead to a unanimous or split decision (Miarka et al., 2017a).

Regarding standing grappling actions, MMA bouts with Unanimous and Split Score Decision outcomes demonstrated higher frequencies of Stand ups/round, Takedowns Attempted and Landed/round than KO/TKO and Submission (Table 2). In addition, the effect size of the statistical analyses showed that these actions were performed more often when the Split score existed, that is, when it was not known which one of the fighters was winning. Different results were observed in a previous study, which demonstrated that ground strikes landed and takedowns landed were the main variables of winning bouts (James et al., 2017). This study presented differences in specific situations, for example Unanimous Decision demonstrated higher takedown attempts than Submission attempts to end the bout. These differences among groups may be related to the application of specific throwing techniques.

According to biomechanical analysis of judo throws, lower extremity techniques executed to the front or side (left/right), such as o-soto-gari and de-ashi-baraí, require high amounts of torque and/or velocity before contact with the opponent, while those executed to the rear orientation that 
involve rotation require more time to be applied (Imamura et al., 2006, 2007). Previous studies on wrestling freestyle demonstrated that leg attacks were the main technical focus when achieving attack efficacy, with 0.27 and $0.34 \mathrm{pts} / \mathrm{min}$, respectively (González et al., 2012; Tünnemann, 2011). Furthermore, lower extremity techniques are generally more successfully incorporated by wider, stronger and heavier athletes (Imamura et al., 2006, 2007; Sacripanti, 2012).

Concerning groundwork grappling actions Submission strategies to end the bout demonstrated higher effect size when compared to $\mathrm{TKO} / \mathrm{KO}$ strategies in Chokes frequency and Locks Attempted/round. One of the more common grappling control positions takes place from the side. From this position, the athlete will stabilize the position and try to exhaust his opponent while looking for and creating opportunities to apply submission techniques or striking techniques. It was noted that no frequencies were recorded for Knee Bar Attempted, possibly because it is not an efficient technique, or because no athletes excelled in this technique. This technique could be an important resource to be trained during grappling sessions.

A potential limitation of performance analysis methods described so far is the reliability of the data entry procedure, or the researcher's ability to reproduce the observed value when the measure is repeated (Gabbett and Mulvey, 2008; Silva et al., 2011; Tabben et al., 2018; Tornello et al., 2013; Slimani et al., 2017). Inter-observer consistency is considered crucial in establishing the reliability of motion analysis systems where the total time, frequency and mean duration of combat actions can exhibit large variations (Hopkins, 2000; Miarka et al., 2014). In the present research, experts carried observations in the same conditions, and performance analyses were substantially correlated, with $94 \%$ of all variables classified as "strong" and 6\% classified as "moderate". The results highlight the good reliability and validity of the present protocol for MMA performance analysis. In conclusion, this study showed a tendency toward specific types of grappling attacks/situation according to the strategy to end the bout. This information can be used by coaches and athletes to develop specific and personalized training programs based on the skills and abilities of the athlete.

\section{Conclusion}

This technical-tactical analysis of UFC ${ }^{\circledR}$ bouts provides novel data useful to researchers, coaches and athletes. The development of technical proficiency and tactical approaches that maximize individual skill proficiency will maximize the athlete's potential for success. The Combat Outcomes demonstrated particular patterns, which can be divided in three large groups i) $\mathrm{KO} / \mathrm{TKO}$, ii) Submissions and iii) Score Decisions (Unanimous and Split) of specific grappling action rates. The grappling action trends consisted of lower values of grappling actions for KO/TKO, higher values in the Submission outcomes for groundwork grappling actions and higher values of standing grappling actions for Score Decisions.

\section{References}

Antoniettô NR, Bello FD, Carrenho Queiroz AC, Berbert de Carvalho PH, Brito CJ, Amtmann J, Miarka B. Suggestions for Professional Mixed Martial Arts Training With Pacing Strategy and Technical-Tactical Actions by Rounds. J Strength Cond Res, 2019; 23: Epub ahead of print.

Brandt R, Bevilacqua GG, Coimbra DR, Pombo LC, Miarka B, Lane AM. Body Weight and Mood State Modifications in Mixed Martial Arts: An Exploratory Pilot. J Strength Cond Res, 2018; 32: 2548-2554

Brito CJ, Miarka B, de Durana ALD, Fukuda DH. Home Advantage in Judo: Analysis by the Combat Phase, Penalties and the Type of Attack. J Hum Kinet, 2017; 22: 213-220

Coswig V, Fukuda DH, Ramos SP, Del Vecchio FB. Biochemical Differences between Official and Simulated Mixed Martial Arts (Mma) Matches. Asian J Sports Med, 2016; 7: e30950 
Coswig VS, Miarka B, Pires DA, da Silva LM, Bartel C, Del Vecchio FB. Weight Regain, But Not Weight Loss, Is Related to Competitive Success in Real-life Mixed Martial Arts Competition. Int J Sport Nutr Exerc Metab, 2018; 14: 1-26

Coswig VS, Ramos SP, Del Vecchio FB. Time-Motion and Biological Responses in Simulated Mixed Martial Arts Sparring Matches. J Strength Cond Res, 2016; 30: 2156-2163

Del Vecchio FB, Hirata SM, Franchini E. A Review of Time-Motion Analysis and Combat Development in Mixed Martial Arts Matches at Regional Level Tournaments. Percept Mot Skills, 2011; 112: 639-648

Fernandes JR, Bello FD, Duarte MAB, Carvalho PHB, Queiroz ACC, Brito CJ, Miarka B. Effect of rule changes on technical-tactical actions correlated with injury incidence in Professional Mixed Martial Arts. J Physic Educ Sport, 2018; 18: 1713 - 1721

Gabbett TJ, Mulvey MJ. Time-Motion Analysis of Small-Sided Training Games and Competition in Elite Women Soccer Players. J Strength Cond Res, 2008; 22: 543-552

Ghoul N, Tabben M, Miarka B, Tourny C, Chamari K, Coquart J. Mixed martial arts induces significant fatigue and muscle damage up to 24 hours post-combat. J Strength Cond Res, 2017; 22

González DEL, Rodriguez AA, Bárcenas MA, Alonso SR. Quantitative Indicators of Technical-Tactical Performance: An Example with Freestyle and Female Top 10 Wrestlers from the 2011 World Senior Championships. Int J Wrest Sci, 2012; 2: 1-24

Gronek P; Wielinski D, Gronek J. Genetic and non-genetic determinants of aggression in combat sports.

Open Life Sci, 2015; 10(1): 7-18

Hopkins WG. Measures of Reliability in Sports Medicine and Science. Sports Med, 2000; 30: 1-15

Hutchison MG, Lawrence DW, Cusimano MD, Schweizer TA. Head Trauma in Mixed Martial Arts. Am J Sports Med, 2014; 42: 1352-1358

Imamura RT, Hreljac A, Escamilla RF, Edwards WB. A Three-Dimensional Analysis of the Center of Mass for Three Different Judo Throwing Techniques. J Sports Sci Med, 2006; 5: 122-131

Imamura RT, Iteya M, Hreljac A, Escamilla RF. A Kinematic Comparison of the Judo Throw Harai-Goshi During Competitive and Non-Competitive Conditions. J Sports Sci Med, 2007; 6: 15-22

James LP, Kelly VG, Beckman EM. Periodization for Mixed Martial Arts. Strength Cond J, 2013; 35: $34-45$

James LP, Robertson S, Haff GG, Beckman EM, Kelly VG. Identifying the Performance Characteristics of a Winning Outcome in Elite Mixed Martial Arts Competition. J Sci Med Sport, 2017; 20: 296-301

Jetton AM, Lawrence MM, Meucci M, Haines TL, Collier SR, Morris DM, Utter AC. Dehydration and Acute Weight Gain in Mixed Martial Arts Fighters before Competition. J Strength Cond Res, 2013; 27: 1322-1326

Karpman S, Reid P, Phillips L, Qin Z, Gross DP. Combative Sports Injuries: An Edmonton Retrospective. Clin J Sport Med, 2016; 26: 332-334

Kirk C, Hurst HT, Atkins S. Measuring the Workload of Mixed Martial Arts Using Accelerometry, Time Motion Analysis and Lactate. Int J Perf Anal Sport, 2015; 15: 359-370

Matthews JJ, Nicholas C. Extreme Rapid Weight Loss and Rapid Weight Gain Observed in UK Mixed Martial Arts Athletes Preparing for Competition. Int J Sport Nutr Exerc Metab, 2016; 27: 122-129

Maszczyk A, Gołaś A, Pietraszewski P, Kowalczyk M, Cięszczyk P, Kochanowicz A, Smółka W, Zając A. Neurofeedback for the enhancement of dynamic balance of judokas. Biol Sport., 2018; 35(1): 99-102

Miarka B, Brito CJ, Amtmann J. Performance probabilities and outcome effects in technical-tactical factors with bout phase changes of mixed martial arts. Int J Perf Anal Sport, 2017; 17: 510-520

Miarka B, Brito CJ, Amtmann J, Córdova C, Bello, DF, Camey S. Suggestions for judo training with pacing strategy and decision making by judo championship phases. J Hum Kinet, 2018; 64: 201-219

Miarka B, Brito CJ, Moreira DG, Amtmann J. Differences by Ending-Rounds and Other Rounds in TimeMotion Analysis of Mixed Martial Arts: Implications for Assessment and Training. J Strength Cond Res, 2018a; 32: 1-27

(c) Editorial Committee of Journal of Human Kinetics 
Miarka B, Coswig V, Brito CJ, Slimani M, Amtmann J, Del Vecchio, FB. Comparison of Combat Outcomes: Technical and Tactical Analysis of Female Mma. Int J Perf Anal Sport, 2016a; 16: 1-13

Miarka B, Coswig VS, Del Vecchio FB, Brito CJ, Amtmann, J. Comparisons of Time-Motion Analysis of Mixed Martial Arts Rounds by Weight Divisions. Int J Perf Anal Sport, 2015; 15: 1-12

Miarka B, Cury R, Julianetti R, Battazza R, Julio UF, Calmet M, Franchini E. A Comparison of Time-Motion and Technical-Tactical Variables between Age Groups of Female Judo Matches. J Sports Sci, 2014; 32: 1529-1538

Miarka B, Dal Bello F, Brito CJ, Del Vecchio FB, Amtmann J, Chamari K. A 12-Year Cohort Study of DocStoppage in Professional Mixed Martial Arts. Int J Sports Physiol Perform, 2019; 14: 606-611

Miarka B, Fukuda DH, Del Vecchio FB, Franchini E. Discriminant Analysis of Technical-Tactical Actions in High-Level Judo Athletes. Int J Perf Anal Sport, 2016b; 16: 30-39

Miarka B, Sterkowicz-Przybycien K, Fukuda DH. Evaluation of Sex-Specific Movement Patterns in Judo Using Probabilistic Neural Networks. Motor Control 2017a; 21: 390-412

Sacripanti A. Biomechanical Revision of the Principles of Dr. Jigoro Kano's Judo Kodokan. Med Del Sport, 2012; 65: 265-281

Santos DAD, Miarka B, Bello FD, Queiroz ACC, de Carvalho PHB, Brito CJ, Beneke R. 10-Years on TimeMotion and Motor Actions of Paired Mixed Martial Arts Athletes. Int J Sports Physiol Perform, 2018; 30: $1-13$

Silva JJR, Del Vecchio FB, Picanço LM, Takito MY, Franchini E. Time-Motion Analysis in Muay-Thai and KickBoxing Amateur Matches. J Hum Sport Exerc, 2011; 6: 490-496

Slimani M, Chaabene H, Miarka B, Chamari K. The Activity Profile of Elite Low-Kick Kickboxing Competition. Int J Sports Physiol Perform, 2017; 12: 182-189

Sterkowicz S, Lech G, Almansba R. The Course of Fight and the Level of Sports Achievements in Judo. Arch Budo, 2007; 3: 72-81

Sterkowicz-Przybycien K, Miarka B, Fukuda DH. Sex and Weight Category Differences in Time-Motion Analysis of Elite Judo Athletes: Implications for Assessment and Training. J Strength Cond Res, 2017; 31: $817-825$

Tabben M, Miarka B, Chamari K, Beneke R. Decisive-moment: A Metric to Determine Success in Elite Karate Bouts. Int J Sports Physiol Perform, 2018; 18:1-18

Tornello F, Capranica L, Chiodo S, Minganti C, Tessitore A. Time-Motion Analysis of Youth Olympic Taekwondo Combats. J Strength Cond Res, 2013; 27: 223-228

Tünnemann H. Analysis of the Wrestling World Championships-Moscow 2010. Int J Wrest Sci, 2011; 1: 67-83

\section{Corresponding author:}

\section{Bianca Miarka}

Av. Eduardo Roberto Daher, n.567

Centro, Itapecerica da Serra - SP,

Brazil. ZIP: 06850-040, Brazil

E-mail: miarkasport@hotmail.com 